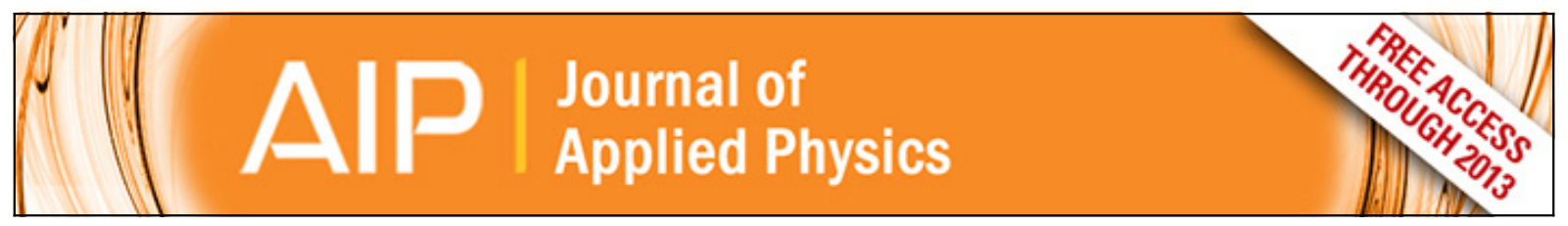

\title{
Single phase nanocrystalline GaMnN thin films with high Mn content
}

S. Granville, F. Budde, B. J. Ruck, H. J. Trodahl, G. V. M. Williams, A. Bittar, M. Ryan, J. Kennedy, A. Markwitz, J. B. Metson, K. E. Prince, J. M. Cairney, and M. C. Ridgway

Citation: Journal of Applied Physics 100, 084310 (2006); doi: 10.1063/1.2357701

View online: http://dx.doi.org/10.1063/1.2357701

View Table of Contents: http://scitation.aip.org/content/aip/journal/jap/100/8?ver=pdfcov

Published by the AIP Publishing

\section{AlP Re-register for Table of Content Alerts}

\section{Create a profile. \\ Sign up today!}




\title{
Single phase nanocrystalline GaMnN thin films with high Mn content
}

\author{
S. Granville, ${ }^{\text {a) }}$ F. Budde, B. J. Ruck, and H. J. Trodahl \\ MacDiarmid Institute for Advanced Materials and Nanotechnology, School of Chemical \\ and Physical Sciences, Victoria University of Wellington, P.O. Box 600, Wellington 6140, New Zealand
}

G. V. M. Williams, A. Bittar, and M. Ryan

Industrial Research Ltd., P.O. Box 31310, Lower Hutt 5040, New Zealand

J. Kennedy and A. Markwitz

National Isotope Centre, GNS Science, 30 Gracefield Road, P.O. Box 31312, Lower Hutt 5040, New Zealand

J. B. Metson

Light Metals Research Centre, The University of Auckland, Private Bag 92019, Auckland 1142, New Zealand

\section{K. E. Prince}

Institute for Nuclear Geophysiology, Australian Nuclear Science and Technology Organisation, Menai, New South Wales 2234, Australia

J. M. Cairney

School of Materials Science and Engineering, University of New South Wales, Sydney, New South Wales 2052, Australia

\author{
M. C. Ridgway \\ Department of Electronic Materials and Engineering, Australian National University, Canberra, \\ Australia Capital Territory 0200, Australia
}

(Received 11 April 2006; accepted 20 July 2006; published online 19 October 2006)

\begin{abstract}
$\mathrm{Ga}_{1-x} \mathrm{Mn}_{x} \mathrm{~N}$ thin films with a Mn content as high as $x=0.18$ have been grown using ion-assisted deposition and a combination of Rutherford backscattering spectroscopy and nuclear reaction analysis was used to determine their composition. The structure of the films was determined from $\mathrm{x}$-ray diffraction, transmission electron microscopy, and extended x-ray absorption fine structure (EXAFS). The films are comprised of nanocrystals of random stacked GaMnN and there is no evidence of Mn-rich secondary phases or clusters. EXAFS measurements at the Mn and Ga edge are almost identical to those at the Ga edge from Mn-free nanocrystalline GaN films, showing that the Mn occupies the Ga lattice sites, and simulated radial distribution functions of possible Mn-rich impurity phases bear no resemblance to the experimental data. The results indicate that these are the most heavily Mn-doped single phase GaN films studied to date. (C) 2006 American Institute of Physics.
\end{abstract}

[DOI: $10.1063 / 1.2357701]$

\section{INTRODUCTION}

The wide-band-gap semiconductor GaN, with a direct energy gap of $3.4 \mathrm{eV}$, is an important material in the development of robust, high-speed optoelectronic devices. Furthermore, GaN doped with magnetic elements such as $\mathrm{Mn},{ }^{1-6}$ $\mathrm{Cr}^{7-9}$ or the rare earths ${ }^{10,11}$ has received much attention because of its potential to allow manipulation of the spin degree of freedom of the charge carriers by introducing a net magnetic moment. Experimental studies of nanocrystalline and amorphous ${ }^{12-14}$ GaN thin films have supported predictions ${ }^{15}$ that even in a heavily disordered state many of the useful properties of the single crystalline material are retained. It is unknown whether the properties of thin films of $\mathrm{GaN}$ with magnetic dopants may also prove resistant to a high degree of disorder.

A wide variety of magnetic behaviors has been reported

${ }^{a)}$ Electronic mail: mrwhereitsat@gmail.com for GaMnN films ${ }^{6,16-20}$ and the exact magnetic response appears to depend strongly on the structure of the material as well as the presence of any ferro- or antiferromagnetic Mnrich impurity phases. ${ }^{21-24}$ A high $\mathrm{Mn}$ concentration in $\mathrm{GaN}$ films is predicted to be necessary for ferromagnetism to persist above room temperature, ${ }^{1}$ but experimental studies on films with $x>5$ at. \% Mn often reveal Mn clustering ${ }^{25-27}$ or secondary phases such as ferrimagnetic $\mathrm{Mn}_{4} \mathrm{~N}$ (Ref. 28) or $\mathrm{Mn}_{3} \mathrm{GaN}^{23,24}$ Investigating the structure of such films is of great importance in determining the origin of the different physical properties reported.

In this paper we report studies of the composition and structure of nanocrystalline GaMnN thin films prepared by ion-assisted deposition (IAD). We have studied the structure of the films using standard X-ray diffraction (XRD) and transmission electron microscopy (TEM) techniques. Extended $\mathrm{x}$-ray absorption fine structure (EXAFS) measurements have also been carried out, allowing for an elementspecific investigation of the short-range $(<5 \AA)$ environment 
TABLE I. Compositions of $\mathrm{Ga}_{1-x} \mathrm{Mn}_{x} \mathrm{~N}$ films in this study as determined by RBS and NRA.

\begin{tabular}{lccccc}
\hline \hline & \multicolumn{5}{c}{ Elemental compositions (at. \%) } \\
\cline { 2 - 6 } & $\mathrm{Ga}$ & $\mathrm{Mn}$ & $\mathrm{N}$ & $\mathrm{O}$ & $\mathrm{C}$ \\
\hline GaMnN5 & 37.7 & 6.4 & 55.3 & 0.5 & $<0.5$ \\
GaMnN9 & 34.5 & 9 & 55.5 & 1 & $<0.5$ \\
\hline \hline
\end{tabular}

around both the Mn and $\mathrm{Ga}$ atoms in the film. EXAFS measurements are an effective complement to XRD and TEM analyses, allowing for the identification of secondary phases not visible in either of the latter two measurements. ${ }^{27}$

\section{EXPERIMENTAL DETAILS}

The IAD gallium manganese nitride thin films reported here were grown at room temperature on silicon, glassy carbon, and polythene film substrates. The use of these different substrate materials facilitates the variety of complementary techniques reported here. We have previously reported the growth of GaN films by IAD, where gallium metal was thermally evaporated onto the substrates in the presence of a beam of $500 \mathrm{eV}$ nitrogen ions from a Kaufmann-type ion source. ${ }^{29}$ To incorporate $\mathrm{Mn}$ into the films, a separate alumina crucible containing Mn flakes was heated at the same time as the Ga. A quartz crystal microbalance shielded from the ion source was used to control the total gallium plus manganese deposition rate, and this was held constant at $1 \AA \mathrm{s}^{-1}$. The relative deposition rates were adjusted so as to vary the final $\mathrm{Ga}: \mathrm{Mn}$ ratio in the films. A second quartz crystal directly adjacent to the substrates measured the actual film growth rate, which was between 0.4 and $0.6 \AA \mathrm{s}^{-1}$. This was less than $1 \AA \mathrm{s}^{-1}$ due to resputtering of $\mathrm{Ga}$ and $\mathrm{Mn}$ atoms by the nitrogen ions. IAD is a nonequilibrium growth method, and we show below that it enables Mn to be incorporated at levels much higher than the normal solubility limit for $\mathrm{GaN}$.

Compositional analysis of the films was made from Rutherford backscattering spectroscopy (RBS) and nuclear reaction analysis (NRA) measurements. The RBS was performed on the films grown on glassy carbon in order to resolve the $\mathrm{N}$ and residual $\mathrm{O}$ backscattered peaks from the substrate, and NRA was performed on the films grown on $\mathrm{Si}$ to determine absolute concentrations of the lighter $\mathrm{N}$ and $\mathrm{O}$. To within the experimental resolution, RBS-derived compositions and thicknesses of films on $\mathrm{Si}$ and glassy carbon substrates resulting from a single preparation are identical. Depth-dependent elemental compositions were further investigated using secondary ion mass spectroscopy (SIMS), performed with a Cameca IMS 5f SIMS microprobe at ANSTO, Lucas Heights, New South Wales, Australia.

XRD spectra of the films were taken using the Co $K \alpha$ line at $1.79 \AA$ at grazing incidence in order to maximize the diffraction peaks from the thin film relative to the substrate material. TEM images were made using a $200 \mathrm{keV}$ Philips CM-200 TEM at the Electron Microscope Unit, University of New South Wales. Both Ga- and Mn-edge EXAFS were performed on the films grown on X-ray transparent polythene

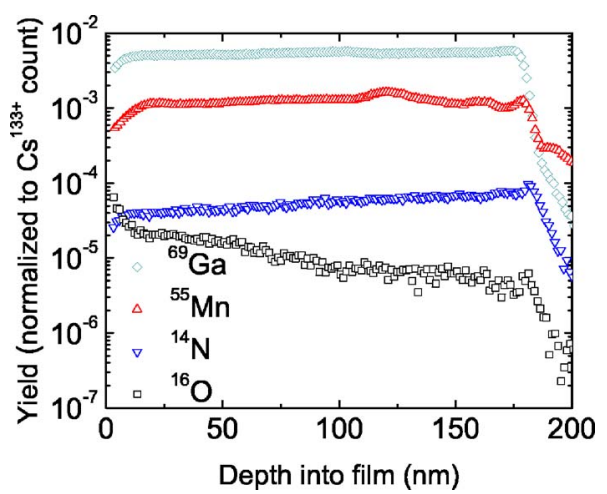

FIG. 1. (Color online) SIMS elemental depth profile of a GaMnN thin film. The Mn and Ga profiles are roughly uniform, and there is a slight increase of $\mathrm{O}$ at the film surface.

substrates on the Australian Synchrotron Beamline Facility at the Photon Factory in Tsukuba, Japan. EXAFS measurements were analyzed and modeled using the IFEFFIT-codebased programs ATHENA and ARTEMIS. ${ }^{30}$

\section{RESULTS AND DISCUSSION}

Table I presents the compositions of the films examined in this paper, as determined by RBS ( $\mathrm{Ga}$ and $\mathrm{Mn}$ ) and NRA $(\mathrm{N}$ and $\mathrm{O})$. The films examined here have $\mathrm{Mn}$ contents of $x=0.13$ and $x=0.18$, making them some of the most heavily $\mathrm{Mn}$-doped GaN films yet reported. The films are $\mathrm{N}$ rich, as is seen in IAD-GaN films, ${ }^{31}$ in which the excess nitrogen is in the form of molecular $\mathrm{N}_{2}$ within the nanocrystalline network. Raman spectroscopy confirms that the excess nitrogen in the present films is also molecular. $\mathrm{O}$ content is at the resolution limit, below 1 at. \%. The SIMS depth profile for the $x$ $=0.18$ film (Fig. 1) shows $\mathrm{Mn}, \mathrm{Ga}$, and $\mathrm{N}$ compositions that are reasonably constant throughout the film, and a slightly O-rich region within $10 \mathrm{~nm}$ of the surface, presumably resulting from postgrowth oxidation.

Figure 2 shows the grazing incidence XRD spectra for the $x=0.18$ film. The spectrum is essentially the same as that from the $x=0.13$ film, with no additional peaks or shifts in their widths or positions. The pattern that appears is very similar to that seen from nanocrystalline IAD-GaN films ${ }^{14}$ and consistent with the same model of random stacked $\mathrm{GaN}$

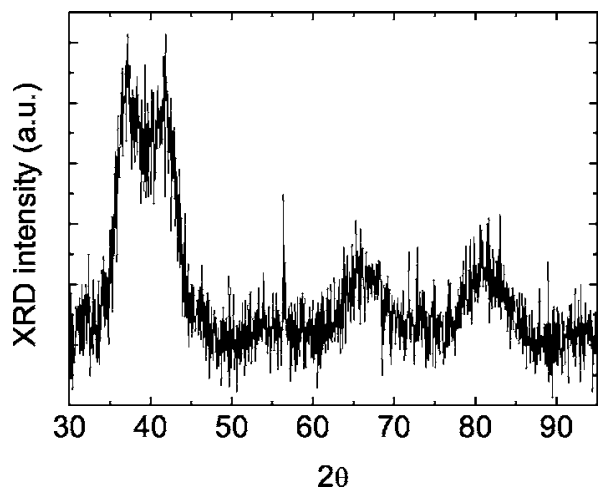

FIG. 2. XRD spectrum from a $200 \mathrm{~nm}$ GaMnN film on Si. The spectrum was taken at grazing incidence $\mathrm{X}$-ray angle to remove sharp scattering peaks from the crystalline substrate. 


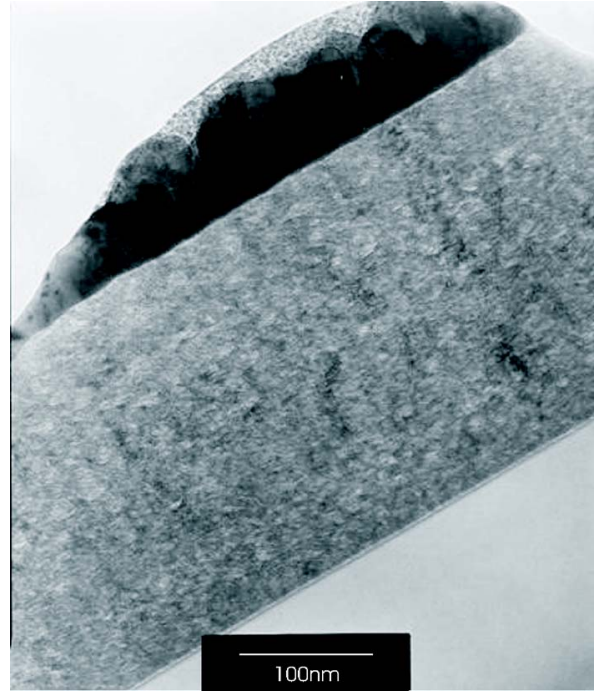

FIG. 3. TEM micrograph of the $\mathrm{Ga}_{0.82} \mathrm{Mn}_{0.18} \mathrm{~N}$ film deposited on a Si substrate. The dark layer at the top of the film is a protective Pt coating layer added before focussed ion beam (FIB) thinning of the film. The measurement bar is shown for scale.

hexagonal planes. A Debye-Scherrer fit to the peak widths yields an average crystallite size of $\sim 3 \mathrm{~nm}$. There is no sign of any secondary crystal phases in the spectra, although the results do not exclude the possibility of a proportion of the $\mathrm{Mn}$ residing in other phases. Small concentrations of nanocrystalline or amorphous Mn-rich secondary phases are often invisible in XRD; however, we will show below through TEM and EXAFS measurements that there is no evidence of secondary phases in these films, and, in particular, EXAFS studies establish that all the $\mathrm{Mn}$ ions are bonded to four $\mathrm{N}$ ions.

TEM imaging can directly identify the presence of nanometer-sized clusters and crystallites within the film. The $x=0.18$ film was thinned to $\sim 100 \mathrm{~nm}$ cross-sectional thickness using a focused ion beam of $\mathrm{Ga}^{+}$atoms. TEM was performed in cross-section through the film, and Fig. 3 shows the bright-field cross-sectional TEM image of the film. The film is uniformly comprised of crystallites, and there is no sign of clusters of Mn as has been seen in other TEM studies

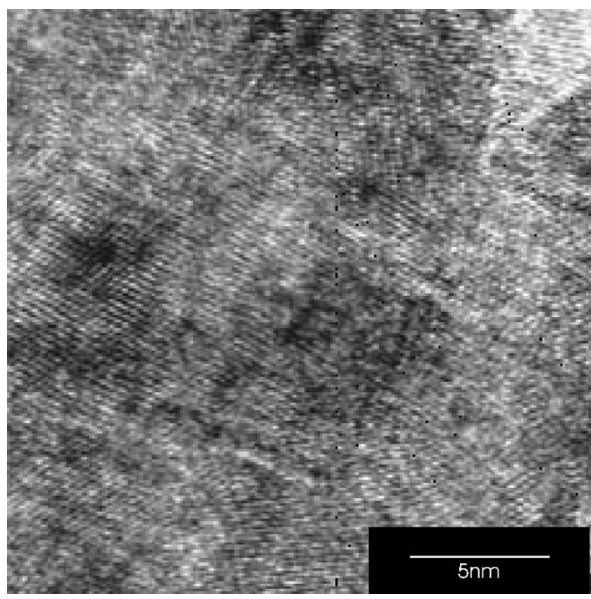

FIG. 4. High-resolution TEM image of a crystallite in the film. The crystallite has sides $\sim 5 \mathrm{~nm}$ long.

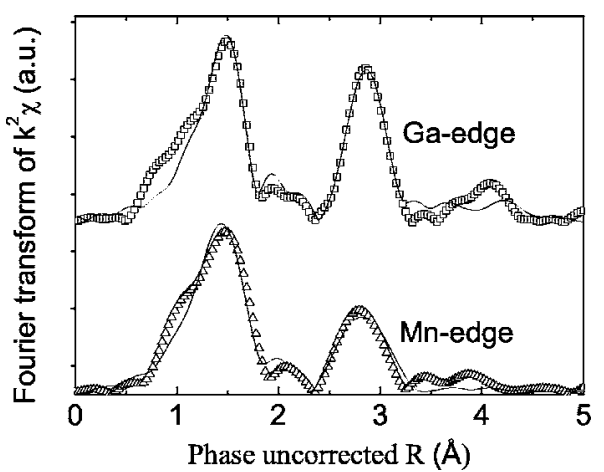

FIG. 5. Fourier transform of EXAFS measurements taken at Ga and Mn edges for the $\mathrm{Ga}_{0.82} \mathrm{Mn}_{0.18} \mathrm{~N}$ film. The squares are the experimental data and the lines are best fits to the data using a model of four $\mathrm{N}$ nearest neighbors and $12 \mathrm{Ga}$ next nearest neighbors. The best fit parameters are printed in Table II.

of GaMnN. ${ }^{25}$ A crystallite is shown at high resolution in Fig. 4 , with a size between 3 and $5 \mathrm{~nm}$, in agreement with the XRD peak widths.

EXAFS measurements were taken at $10 \mathrm{~K}$ at both $\mathrm{Ga}$ $(10363 \mathrm{eV})$ and $\mathrm{Mn}(6550 \mathrm{eV}) \mathrm{K}$ absorption edges for each sample. The Fourier transforms of the spectra taken in photoelectron wave vector space are pseudo-radial-distributionfunctions, and are used to determine the radii of shells of atoms around the absorbing atom of interest. In Fig. 5, the Fourier transforms of both the $\mathrm{Ga}$ and $\mathrm{Mn}$ edges of the $x$ $=0.18$ sample are plotted. The first two neighboring peaks (corresponding primarily to $\mathrm{Ga}-\mathrm{N}, \mathrm{Ga}-\mathrm{Ga}$ for the $\mathrm{Ga}$ edge or $\mathrm{Mn}-\mathrm{N}, \mathrm{Mn}-\mathrm{Ga}$ for the Mn edge) are at the same radial distance from each scattering atom, suggesting that the local environment around each atom is very similar. The Fourier transformed spectra for both edges closely resemble that of the Ga edge in Mn-free $n x$-GaN EXAFS. ${ }^{14}$ A fit was made to the first two peaks from both edges using a fixed coordination of four $\mathrm{N}$ nearest neighbor atoms and $12 \mathrm{Ga}$ next nearest neighbor atoms, features common to wurtzite, zinc blende, or random-stacked GaN sequences. The fitted values for the model were shell radius $(R)$, Debye-Waller disorder factor $\left(\sigma^{2}\right)$, and edge energy correction factor $\left(E_{0}\right)$. The best fit parameters are reported in Table II. Note that wurtzite GaN actually has three nearest neighbor nitrogen atoms at $1.946 \AA$ and one nearest neighbor nitrogen atom at a reduced distance of $1.937 \AA$. However, this difference is below the uncertainty in our measured bond length $( \pm 0.01 \AA)$.

The radial distances to the first and second nearest shells from the $\mathrm{Mn}$ and $\mathrm{Ga}$ edges are equal to within the fitting

TABLE II. EXAFS parameters for a model of wurtzite GaN fitted to the $\mathrm{Ga}$ and $\mathrm{Mn} K$ edges of $\mathrm{Ga}_{0.82} \mathrm{Mn}_{0.18} \mathrm{~N}$.

\begin{tabular}{lcccc}
\hline \hline & $\begin{array}{c}\text { Nearest } \\
\text { neighbor } \\
\text { atom }\end{array}$ & $\begin{array}{c}R \\
(\AA)\end{array}$ & $\begin{array}{c}\sigma^{2} \\
\left(10^{-3} \AA^{2}\right)\end{array}$ & $\begin{array}{c}\Delta E \\
(\mathrm{eV})\end{array}$ \\
\hline Ga edge & $\mathrm{N}$ & $1.92 \pm 0.01$ & $2.8 \pm 1.3$ & $4.23 \pm 0.64$ \\
& $\mathrm{Ga}$ & $3.22 \pm 0.01$ & $9.8 \pm 0.9$ & $10.97 \pm 2.04$ \\
Mn edge & $\mathrm{N}$ & $1.93 \pm 0.02$ & $5.6 \pm 3.0$ & $1.82 \pm 2.12$ \\
& $\mathrm{Ga}$ & $3.19 \pm 0.03$ & $17.7 \pm 2.8$ & $5.52 \pm 2.27$ \\
\hline \hline
\end{tabular}




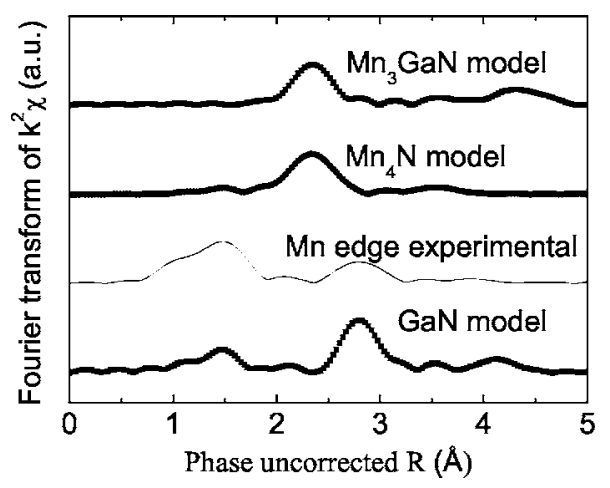

FIG. 6. Simulations of the pseudo-radial-distribution-functions for $\mathrm{Mn}_{3} \mathrm{GaN}, \mathrm{Mn}_{4} \mathrm{~N}$, and $\mathrm{GaN}$ and the Fourier-transformed Mn edge experimental data from the $\mathrm{Ga}_{0.82} \mathrm{Mn}_{0.18} \mathrm{~N}$ film. The experimental data are significantly different from the Mn-containing phases, but closely resemble the GaN simulation.

uncertainty, and both agree with the shell radii around $\mathrm{Ga}$ in Mn-free crystalline GaN. This result contrasts with EXAFS measurements on single-crystalline GaMnN films that exhibit a lengthening of the $\mathrm{Mn}-\mathrm{N}$ bond by almost $5 \%$ for $x$ $=0.057 .^{32,33}$ An increase of this magnitude would be easily signaled in the data of Table II, so we conclude that the structure includes Mn primarily in sites substituting for Ga, and with similar bond lengths. The values of $\sigma^{2}$ that we report are larger than values reported from previous EXAFS studies on single-crystalline $\mathrm{GaMnN},{ }^{32,35}$ implying that the range of bond lengths is large for both ionic species in our films. Since these measurements were performed at low temperature, thermal effects on this disorder parameter are expected to be small. This indicates the displacement is due primarily to intrinsic structural disorder in the films that is greater than in single crystal films, even within the first atomic shell. The combination of the IAD growth process and the very high Mn content results in films far from their lowest energy state, evidently encouraging substitutional Mn. We note in this regard that there is a slight increase of the $\mathrm{Mn}-\mathrm{N}$ bond length in a nanocrystalline $\mathrm{GaMnN}$ film that we have annealed, with a corresponding decrease of bond length mean-square displacement $\left(\sigma^{2}\right) .^{34}$

Plotted in Fig. 6 are the Fourier transforms for EXAFS spectra calculated from models of two impurity phases reported in other studies of $\mathrm{GaMnN}, \mathrm{Mn}_{4} \mathrm{~N}$, and $\mathrm{Mn}_{3} \mathrm{GaN}$, as well as a model of undoped wurtzite GaN, compared with the Mn-edge experimental data. The presence of a strongly scattering Mn-related peak at a phase-uncorrected radius of $\sim 2.3 \AA$ in the two Mn-rich compounds does not correlate with the Fourier transforms from the experimental data for either edge, whereas the wurtzite GaN pseudo-radialdistribution-function closely matches the experimental data. The measurements strongly suggest that the $\mathrm{Mn}$ is predominantly incorporated into the film substitutionally for the $\mathrm{Ga}$ atoms rather than forming clusters or nanocrystals of $\mathrm{Mn}$ rich phases. The $\mathrm{Mn}$ in these GaMnN films is then bonded in the same way as $\mathrm{Ga}$ in $\mathrm{GaN}$ films, tetrahedrally bonded to four $\mathrm{N}$ atoms.

The magnetic character of these films has been examined and they are found to show no sign of ferromagnetism to $5 \mathrm{~K}$. A fuller investigation of the magnetic behavior of IAD$\mathrm{GaMnN}$ films will be published elsewhere.

\section{CONCLUSIONS}

The structure and composition of $\mathrm{GaMnN}$ thin films grown using ion-assisted deposition have been investigated. The Mn content of the films is up to $x=0.18$. The films are found to be nanocrystalline, with a model of a randomly ordered stacking sequence qualitatively accounting for the peaks in the XRD spectra. SIMS, XRD, and TEM measurements suggest that the films are homogeneous, with no signs of Mn clustering or impurity phases as seen in single crystalline films with $\mathrm{Mn}$ concentrations higher than $x \sim 0.05$. EXAFS measurements are consistent with the Mn replacing $\mathrm{Ga}$ atoms substitutionally and show no sign of secondary phases often seen in other recent studies. There is a suggestion that the high levels of Mn doping and the nonequilibrium growth process result in films with a considerable strain in the first atomic shell.

\section{ACKNOWLEDGMENTS}

The authors gratefully acknowledge financial support from the New Zealand Foundation for Research Science and Technology through its New Economy Research Fund, and through a postdoctoral fellowship of one of the authors (B.J.R.). The work of the MacDiarmid Institute is supported by a New Zealand Centre of Research Excellence award. Another author (S.G.) wishes to thank Education New Zealand for financial support of the EXAFS measurements.

${ }^{1}$ T. Dietl, H. Ohno, F. Matsukura, J. Cibert, and D. Ferrand, Science 287, 1019 (2000).

${ }^{2}$ G. P. Das, B. K. Rao, and P. Jena, Phys. Rev. B 68, 035207 (2003).

${ }^{3}$ G. T. Thaler, M. E. Overberg, B. Gila, R. Frazier, C. R. Abernathy, S. J. Pearton, J. S. Lee, S. Y. Lee, Y. D. Park, Z. G. Khim, J. Kim, and F. Ren, Appl. Phys. Lett. 80, 3964 (2002).

${ }^{4}$ K. Huh, M. Ham, J. Myoung, J. Lee, K. Lee, J. Chang, S. Han, H. Kim, and W. Lee, Jpn. J. Appl. Phys., Part 2 41, L1069 (2002).

${ }^{5}$ J. M. Baik, J. Lee, Y. Shon, and T. W. Kang, J. Appl. Phys. 93, 9024 (2003).

${ }^{6}$ M. Zając, J. Gosk, M. Kamińska, A. Twardowski, T. Szyszko, and S. Posiadło, Appl. Phys. Lett. 79, 2432 (2001).

${ }^{7}$ X. Y. Cui, J. E. Medvedeva, B. Delley, A. J. Freeman, N. Newman, and C. Stampfl, Phys. Rev. Lett. 95, 256404 (2005).

${ }^{8}$ S. E. Park, H. Lee, Y. C. Cho, S. Jeong, C. R. Cho, and S. Cho, Appl. Phys. Lett. 80, 4187 (2002).

${ }^{9}$ J. J. Kim, H. Makino, K. Yamazaki, A. Ino, H. Namatame, M. Taniguchi, T. Hanada, M. W. Cho, and T. Yao, Curr. Appl. Phys. 4, 603 (2004).

${ }^{10}$ N. Teraguchi, A. Suzuki, Y. Nanishi, Y. Zhou, M. Hashimoto, and H. Asahi, Solid State Commun. 122, 651 (2002).

${ }^{11}$ S. Dhar, O. Brandt, M. Ramsteiner, V. F. Sapega, and K. H. Ploog, Phys. Rev. Lett. 94, 037205 (2005).

${ }^{12}$ A. Bittar, H. J. Trodahl, N. T. Kemp, and A. Markwitz, Appl. Phys. Lett. 78, 619 (2001).

${ }^{13}$ Y. Kang and D. C. Ingram, J. Appl. Phys. 93, 3954 (2003).

${ }^{14}$ F. Budde et al., J. Appl. Phys. 98, 063514 (2005).

${ }^{15}$ P. Stumm and D. A. Drabold, Phys. Rev. Lett. 79, 677 (1997).

${ }^{16}$ M. L. Reed, N. A. El-Masry, H. H. Stadelmaier, M. K. Ritums, M. J. Reed, C. A. Parker, J. C. Roberts, and S. M. Bedair, Appl. Phys. Lett. 79, 3473 (2001).

${ }^{17}$ Y. Shon, Y. H. Kwon, D. Y. Kim, X. Fan, D. Fu, and T. W. Kang, Jpn. J. Appl. Phys., Part 1 40, 5304 (2001).

${ }^{18}$ S. Sonoda, S. Shimizu, T. Sasaki, Y. Yamamoto, and H. Hori, J. Cryst. Growth 237-239, 1358 (2002). 
${ }^{19}$ K. H. Kim et al., J. Appl. Phys. 93, 6793 (2003).

${ }^{20}$ S. Dhar, O. Brandt, A. Trampert, K. J. Friedland, Y. J. Sun, and K. H. Ploog, Phys. Rev. B 67, 165205 (2003)

${ }^{21}$ S. J. Pearton et al., J. Phys.: Condens. Matter 16, R209 (2004).

${ }^{22}$ K. J. Lee, F. C. Yu, J. A. Kim, D. J. Kim, B. G. Kim, Y. H. Kang, H. J. Kim, and Y. E. Ihm, Phys. Status Solidi B 241, 1525 (2004).

${ }^{23}$ K. H. Kim et al., Appl. Phys. Lett. 82, 1775 (2003).

${ }^{24} \mathrm{R}$. Giraud et al., Europhys. Lett. 65, 553 (2004).

${ }^{25}$ S. Dhar et al., Appl. Phys. Lett. 82, 2077 (2003).

${ }^{26}$ J. M. Baik, H. S. Kim, C. G. Park, and J. Lee, Appl. Phys. Lett. 83, 2632 (2003).

${ }^{27}$ B. He et al., Appl. Phys. Lett. 88, 051905 (2006).
${ }^{28}$ S. S. A. Seo et al., Appl. Phys. Lett. 82, 4749 (2003).

${ }^{29}$ H. J. Trodahl, F. Budde, B. J. Ruck, S. Granville, A. Koo, and A. Bittar, J. Appl. Phys. 97, 084309 (2005).

${ }^{30}$ B. Ravel and M. Newville, J. Synchrotron Radiat. 12, 537 (2005).

${ }^{31}$ B. J. Ruck et al., Phys. Rev. B 70, 235202 (2004).

${ }^{32}$ R. Bacewicz, J. Filipowicz, S. Podsiadło, T. Szyszko, and M. Kamiński, J. Phys. Chem. Solids 64, 1469 (2003).

${ }^{33}$ M. Sato, H. Tanida, K. Kato, T. Sasaki, Y. Yamamoto, S. Sonoda, S. Shimizu, and H. Hori, Jpn. J. Appl. Phys., Part 1 41, 4513 (2002).

${ }^{34}$ A. Koo, Ph.D. thesis, Victoria University of Wellington, 2005.

${ }^{35} \mathrm{X}$. Biquard, O. Proux, J. Cibert, D. Ferrand, H. Mariette, R. Giraud, and B. Barbara, J. Supercond. 16, 127 (2003). 